\title{
Coherent and robust high-fidelity generation of a biexciton in a quantum dot by rapid adiabatic passage
}

\author{
Timo Kaldewey, ${ }^{1, *}$ Sebastian Lüker, ${ }^{2}$ Andreas V. Kuhlmann, ${ }^{1,3}$ Sascha R. Valentin, ${ }^{4}$ Arne Ludwig, ${ }^{4}$ Andreas D. Wieck, ${ }^{4}$ \\ Doris E. Reiter, ${ }^{2}$ Tilmann Kuhn, ${ }^{2}$ and Richard J. Warburton ${ }^{1}$ \\ ${ }^{1}$ Department of Physics, University of Basel, Klingelbergstrasse 82, CH-4056 Basel, Switzerland \\ ${ }^{2}$ Institut für Festkörpertheorie, Universität Münster, Wilhelm-Klemm-Strasse 10, D-48149 Münster, Germany \\ ${ }^{3}$ IBM Research-Zurich, Säumerstrasse 4, 8803 Rüschlikon, Switzerland \\ ${ }^{4}$ Lehrstuhl für Angewandte Festkörperphysik, Ruhr-Universität Bochum, D-44780 Bochum, Germany
}

(Received 2 December 2016; published 10 April 2017)

\begin{abstract}
A biexciton in a semiconductor quantum dot is a source of polarization-entangled photons with high potential for implementation in scalable systems. Several approaches for nonresonant, resonant, and quasiresonant biexciton preparation exist, but all have their own disadvantages; for instance, low fidelity, timing jitter, incoherence, or sensitivity to experimental parameters. We demonstrate a coherent and robust technique to generate a biexciton in an InGaAs quantum dot with a fidelity close to 1 . The main concept is the application of rapid adiabatic passage to the ground-state-exciton-biexciton system. We reinforce our experimental results with simulations which include a microscopic coupling to phonons.
\end{abstract}

DOI: 10.1103/PhysRevB.95.161302

Entangled photon pairs are a powerful resource, especially for quantum teleportation and quantum key distribution protocols. Spontaneous parametric down-conversion in nonlinear optics is a source of entangled photon pairs [1], but success is not guaranteed - the emission is a probabilistic process - and the error rate is high. In contrast, semiconductor quantum dots (QDs) are bright, on-demand sources of both single photons [2] and entangled photon pairs and hence have enormous potential in quantum computing and quantum cryptography [3].

A biexciton in a QD is the starting point for a two-photon cascade: when perfectly prepared, biexciton decay leads to the subsequent emission of two photons [Fig. 1(f)]. In a QD without a significant fine-structure splitting (FSS), the two photons are polarization entangled [4]. The majority of InGaAs QDs show a FSS due to a reduced symmetry [5-7]. However, sophisticated techniques were developed to compensate for the FSS with strain [8], electric [9], or magnetic fields [10,11] and with special growth conditions [7].

Several approaches for biexciton preparation have been proposed [12-15] and demonstrated [4,16-20]. Resonant two-photon schemes involving Rabi rotations [4,17,18] are sensitive to fluctuations in both laser power and QD optical frequency. They are likely to suffer from an imperfect biexciton preparation resulting in undesired exciton photons unrelated to the cascade process.

A more robust scheme using phonon-assisted excitation was reported by several groups recently [18-22]. An impressively high biexciton occupation of up to $95 \%$ was demonstrated using this quasiresonant scheme [20]. But the strength here is also a weakness. The scheme relies on the coupling to the phonon bath in the semiconductor environment: it is an inherently incoherent process. Also, a dependence on relaxation processes in the state preparation results in a timing jitter. In some cases, charge-carrier relaxation times can reach values of up to a nanosecond [23].

We present here a coherent technique to create a biexciton with high probability, low jitter, and weak dependence on the

*timo.kaldewey@unibas.ch excitation and system parameters. The technique is based on rapid adiabatic passage (RAP). RAP allows the robust creation of an exciton [24-26] via a process requiring two levels. RAP is applied here to the ground-state-exciton-biexciton system, $|0\rangle-\left|X^{0}\right\rangle-\left|2 X^{0}\right\rangle$, a three-level system [12,15], and allows biexciton creation without significant exciton creation. In the implementation here, we use the full bandwidth of ultrashort 130 fs pulses allowing us to access spectrally both the groundstate-exciton and exciton-biexciton transitions within one laser pulse. The broad bandwidth pulses have the advantage over the narrow bandwidth pulses suggested in [12] of enhanced robustness owing to stronger avoided crossings and a decoupling of phonons even for negative chirped pulses. We describe this process theoretically and demonstrate excellent agreement with the experimental results. Moreover, we analyze the influence of phonons on the preparation protocol. For RAP-based exciton creation, the influence of phonons depends sensitively on the sign of the chirp [26-28]. We find also in RAP-based biexciton creation that we can choose the chirp such that the phonons are unimportant at low temperature.

We study self-assembled InGaAs QDs at a temperature of 4.2 K. The QDs are grown by molecular-beam epitaxy and embedded in an $n-i-p$ or $n-i$-Schottky structure [Fig. 1(a)] (with more details in the Supplemental Material (SM) [29]). The bias voltage allows control over the QD charge via Coulomb blockade; and within a charging plateau, control over the optical resonance frequency via the dc Stark shift [Fig. 1(d)]. The biexciton binding energy is positive and in the order of a few meV, a typical feature for InGaAs QDs. On driving the optical resonance with a narrow-band continuouswave laser and detecting the resonance fluorescence (RF), we find that QDs in both samples have linewidths below $2 \mu \mathrm{eV}$ [Fig. 1(c)], close to the transform limit [30]. Above saturation on a QD in the $n-i-p$ sample [Fig. 1(a)] we detect a RF single-photon count rate of $5 \mathrm{MHz}$ [Fig. 1(b)]. In the RAP experiments, we excite single QDs with the full bandwidth of $130 \mathrm{fs}$ pulses with a center wavelength of around $940 \mathrm{~nm}$ and linear polarization. The spectral full width at half maximum (FWHM) of the pulses is $\Delta \lambda=10 \mathrm{~nm}$. This allows us to address the exciton and biexciton optical transitions (but 


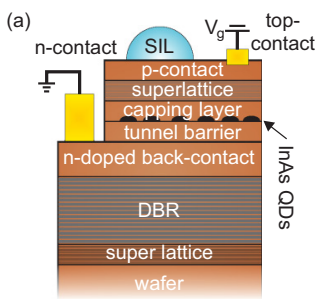

(b)

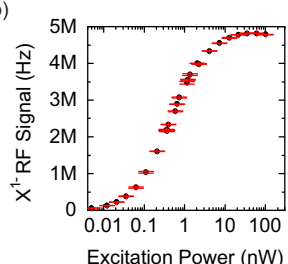

(c)
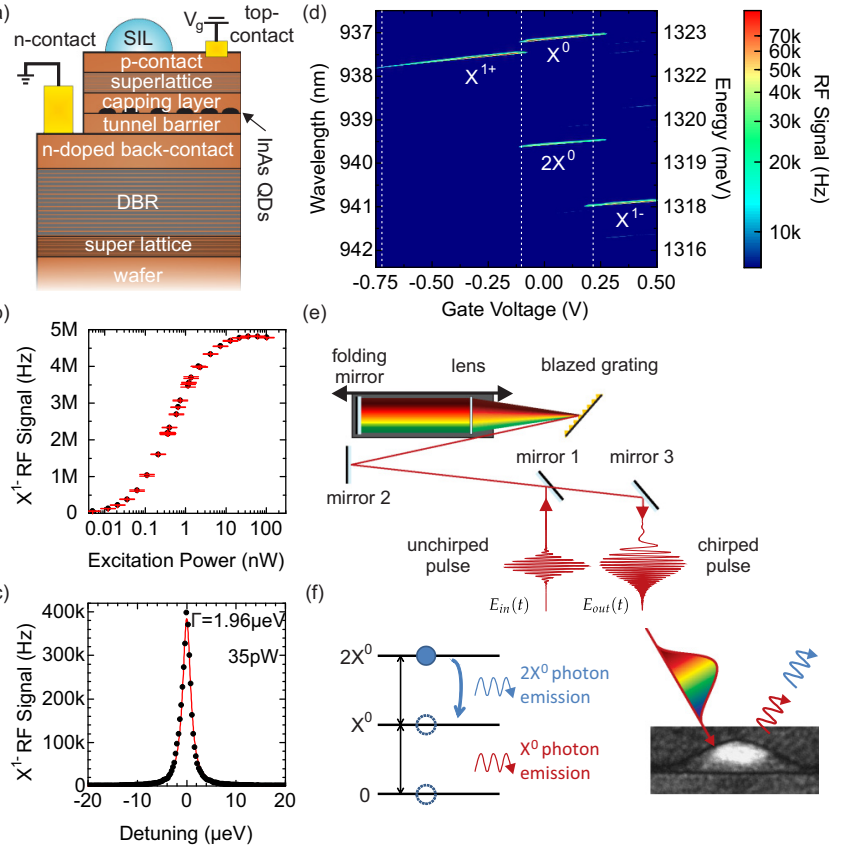

(e)

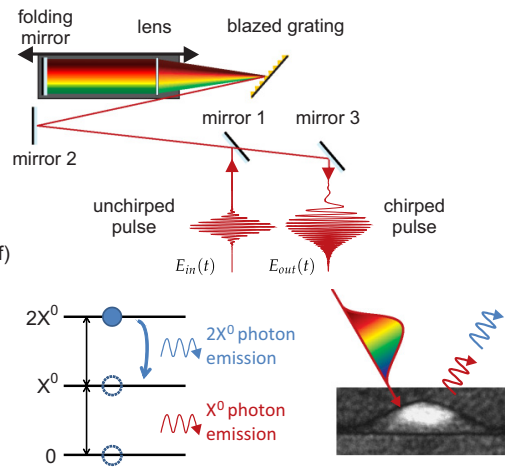

FIG. 1. Resonance fluorescence (RF) on a single quantum dot excited with a narrow-band continuous wave laser and with broadband laser pulses. (a) Structure of the $n-i-p$ diode with epitaxial gate. (b), (c) RF with narrow-band excitation. (b) $X^{1-}$ RF signal versus excitation power. (c) RF versus laser detuning at an excitation power of $35 \mathrm{pW}$ (points). A Lorentzian fit (red solid line) determined the FWHM linewidth, $1.96 \mu \mathrm{eV}$. (d) RF with broadband pulsed excitation as a function of gate voltage. The laser polarization was linear, the chirp positive, and the center frequency resonant with the neutral exciton $X^{0}$. (e) Scheme of a folded $4 f$ pulse shaper controlling the chirp introduced into an ultrashort, transform-limited laser pulse. (f) Generation of a biexciton with broadband, chirped excitation. Scheme of the two-photon cascade (left) after chirped excitation of a QD (right).

not the transitions involving higher shells in the QD) with just one laser pulse. The transform-limited pulses (repetition rate of $76 \mathrm{MHz}$ ) from a passively mode-locked laser were manipulated in a folded $4 f$ pulse shaper [31] [Fig. 1(e)] in order to introduce chirp [29]. We control precisely the sign and magnitude of the chirp [29]. The FWHM of the pulse duration in intensity is stretched up to $\Delta t=15 \mathrm{ps}$ covering chirp coefficients up to $|\alpha|=0.70 \mathrm{ps}^{2}$. For more details about the setup, we refer to the SM [29].

The electronic structure in QDs is in general complex. For example, in II-VI semiconductor colloidal QDs the electronic structure of multiexciton complexes was studied [32] and several biexciton states were identified [33]. However, in the III-V semiconductor QDs studied here, only the $s$ shells are populated such that the system can be described by four states, namely, the ground state $|0\rangle$, the two bright exciton states $\left|X_{H / V}^{0}\right\rangle$, and a single biexciton $\left|2 X^{0}\right\rangle$ [Fig. 2(a)]. The $p$ shells are energetically far removed even when using the full bandwidth of the ultrafast laser pulses. The energy of the biexciton state $\left|2 X^{0}\right\rangle$ is $\hbar \omega_{2 X^{0}}=2 \hbar \omega_{X^{0}}-\Delta_{B}$ where $\hbar \omega_{X^{0}}$ is the energy of the neutral exciton and $\Delta_{B}$ the biexciton binding energy. These energies are determined experimentally from the (a)
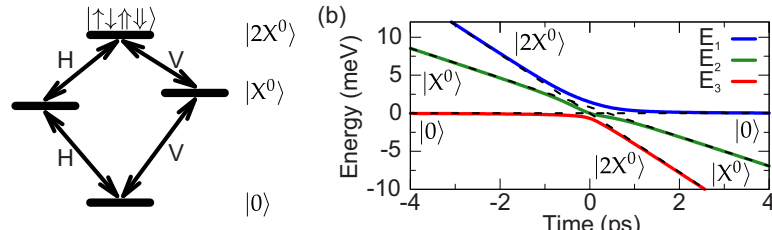

(c)

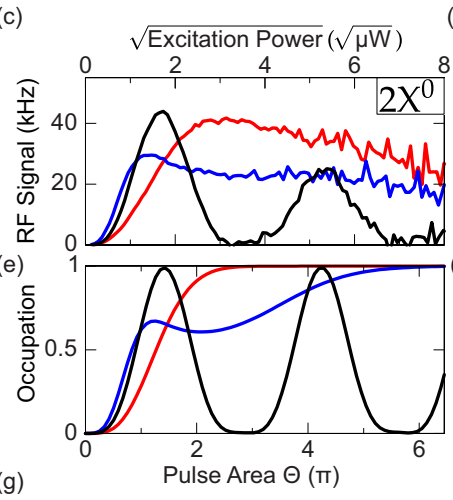

(d)
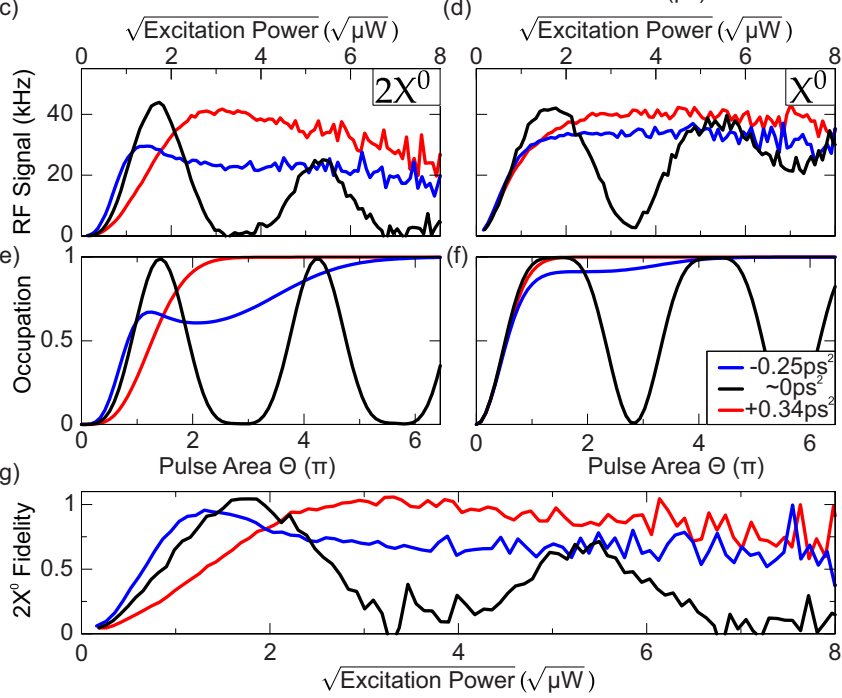

FIG. 2. Biexciton generation on QD1. (a) Energy level scheme. (b) Instantaneous eigenenergies, the dressed states. (c) $2 X^{0}$ emission and (d) $X^{0}$ emission as a function of the time-averaged square-root excitation power for different chirp parameters. (e), (f) Simulation of the biexciton occupation and the sum of exciton and biexciton occupations as a function of the pulse area. (g) Ratio of the experimental data: $2 X^{0} / X^{0}$.

emission spectrum. Considering only one linear polarization, $H$ or $V$, and assuming that the laser pulses are much faster than the fine-structure-induced quantum beat, a three-level system with only one exciton state, $\left|X^{0}\right\rangle$, is sufficient.

The exciton and biexciton states are coupled to longitudinal acoustic phonons in the pure dephasing regime via the deformation potential [29]. We use standard GaAs parameters and take the excitation parameters from the experiment leaving the QD size as the only fitting parameter. This model has been successfully used to describe the phonon influence on Rabi rotations [34] and on the population inversion via RAP $[12,27]$. For the occupations of the exciton and biexciton states as well as for the coherences between all involved states a set of equations of motion is derived within a well-established fourth-order correlation expansion method [35]. The equations are then solved numerically.

The goal of our work is high-fidelity biexciton generation using RAP. Ideally, a vanishing intermediate occupation of the exciton is required. Less critical is a residual occupation of the ground state: this reduces only the rate of entangled photon emission but not the entanglement. A figure of merit is the ratio of biexciton photons to exciton photons. The ideal case is a ratio of one where every exciton photon is part of the two-photon cascade on biexciton decay [Fig. 1(f)]. In determining a biexciton creation fidelity we assume that biexciton and exciton photons are created and detected with equal probability. This is likely to be a very good assumption. 
The biexciton could in principle decay by an Auger process but in practice the probability of an Auger process is very small (probability of $0.23 \%$ [36]); and a reexcitation process during the cascade decay is also very unlikely on account of the hierarchy of times [the pulse duration (up to $15 \mathrm{ps}$ ) is much shorter than the radiative lifetime (1 ns) which is much shorter than the time separating successive pulses (13 ns)]. Therefore, we define here the fidelity as the ratio of detected photons from the transition $\left|2 X^{0}\right\rangle \rightarrow\left|X^{0}\right\rangle\left(2 X^{0}\right.$ photons $)$ and from the transition $\left|X^{0}\right\rangle \rightarrow|0\rangle$ ( $X^{0}$ photons).

Figure 2 shows data from a single QD in the $n$ - $i$-Schottky device. The laser pulses are centered at the two-photon biexciton resonance at $944.3 \mathrm{~nm}$. The $2 X^{0}$ and $X^{0}$ emission intensities and their ratio are shown as a function of the square root of the excitation power in Figs. 2(c), 2(d) and 2(g), respectively. For close-to-zero chirp (black curves in Fig. 2), Rabi rotations are observed. At the first maximum, the $X^{0}$ and $2 X^{0}$ emission intensities are equal to within an error of $5 \%$, then both curves go down nearly to zero. At the second maximum, the $2 X^{0}$ emission reduces to $75 \%$ of the $X^{0}$ emission. For higher excitation power, we observe mainly $X^{0}$ emission with little emission from the $2 X^{0}$. At the first maximum it is clear that ultrafast pulses with close-to-zero chirp enable high-fidelity preparation of the biexciton by a Rabi rotation. However, this $\pi$-pulse excitation is very sensitive to variations in the detuning or excitation power. We turn to RAP which potentially offers a more robust scheme.

To create the biexciton using RAP, we first concentrate on positive chirp. Introducing a chirp of $+0.34 \mathrm{ps}^{2}$ stretches the pulse to an intensity FWHM of 8 ps. In the experiment, the $2 X^{0}$ emission rises more slowly with pulse power than in the Rabi rotation experiment (red curve in Fig. 2) but then reaches a very broad maximum. Both $2 X^{0}$ and $X^{0}$ signals reach a common maximum where the signals correspond closely to the maximum achieved in the Rabi rotation experiment. These are the main signatures of RAP. In terms of $2 X^{0}$ preparation, the ratio of $2 X^{0}$ to $X^{0}$ emission reaches the ideal case of $100 \%$ with an error of $5 \%$, proving that we can achieve a high-fidelity biexciton preparation with RAP, robust against power and detuning fluctuations.

We now focus on the response of the system to a negative chirp of $-0.25 \mathrm{ps}^{2}$ (pulse duration of $6 \mathrm{ps}$, blue curves in Fig. 2). In this case the $2 X^{0}$ signal rises at smaller pulse areas than for positive chirp and in fact at even smaller pulse areas than the Rabi rotations. The maximum of the $2 X^{0}$ signal is considerably less than the signal following a $\pi$ Rabi pulse. The maximum $X^{0}$ signal is likewise reduced. The highest $2 X^{0}$ to $X^{0}$ ratio is $94 \%$ decreasing rapidly at high pulse areas. A negative chirp works less well at $2 X^{0}$ creation than a positive chirp. However, at higher excitation powers the difference between positive and negative chirp becomes less and less pronounced.

An analysis of the calculated instantaneous eigenenergies, the dressed states Fig. 2(b), allows a qualitative understanding of the physics. Starting in the ground state $|0\rangle$, the system evolves along the lower (red) branch for positive chirp. Provided that the pulse area is above the RAP threshold, the system remains in the lower branch at the $|0\rangle$ and $\left|2 X^{0}\right\rangle$ avoided crossing such that the system ends up in the biexciton state $\left|2 X^{0}\right\rangle$. Although there is no direct coupling between the $|0\rangle$ and $\left|2 X^{0}\right\rangle$ states, the avoided crossing arises as $|0\rangle$ and $\left|2 X^{0}\right\rangle$ have a common coupling to the $\left|X^{0}\right\rangle$ state. Phonons can interrupt the adiabatic transfer by causing a jump from one branch to the other [12]. For positive chirp, the system starts in the lowest-energy dressed state and only phonon absorption is possible. At $4.2 \mathrm{~K}$, however, phonons at the relevant energy scale are frozen out and the probability for absorption is small. Hence the process with positive chirp is barely affected by phonons at low temperature. Conversely, for negative chirp the system starts out in the uppermost branch [blue curve in Fig. 2(b) with time running from "right" to "left"]; the system can now jump to lower branches by phonon emission, a process which is possible even at low temperature. This leads to a significant probability of exciton and ground-state population, reducing the fidelity of the RAP process. All these observations correspond well to the experimental data.

The theory provides a quantitative account of three-level RAP in the presence of phonon coupling. We calculate the $2 X^{0}$ and $X^{0}$ occupations as a function of the pulse area: the $2 X^{0}$ signal is proportional to the $2 X^{0}$ occupation; the $X^{0}$ signal is proportional to the sum of the $2 X^{0}$ and $X^{0}$ occupations. The simulation results are also shown in Fig. 2 and reproduce the main features of the experimental data extremely well, notably the exact form of the Rabi oscillations for close-to-zero chirp (a broad minimum at pulse area $\sim 3 \pi$ in the $2 X^{0}$ signal, a broad maximum at $\sim 1.5 \pi$ in the $X^{0}$ signal); the "delayed" ("accelerated") rise of the $2 X^{0}$ signal for positive (negative) chirp; the relative signal strengths; and the better RAP performance for positive chirp. In the theory, the Rabi rotations are not influenced significantly by phonons because the Rabi dynamics are too fast for the phonons to follow. The theoretical results show clearly that $2 X^{0}$ generation with RAP is strongly (weakly) influenced by phonons for negative (positive) chirp for small to modest pulse areas. At the highest pulse areas, however, the theory predicts essentially perfect RAP independent of the sign of the chirp. The interpretation is that the splittings between the branches at the avoided crossings are energetically so large that they lie well above the energy range of the phonons which are efficiently coupled to the exciton system [34]. In other words, the wavelength of the phonons required for scattering between the dressed states becomes much smaller than the size of the QD at high pulse area. There is evidence that this reduction of the phonon efficiency is also seen in the experiment: the RAP signals for positive and negative chirp approach each other at the largest pulse areas. However, in addition, the experimental signals exhibit a decay at high pulse areas which cannot be explained by the phonon coupling model. Instead, we tentatively attribute this decay to an occupation of higher-energy levels by multiphoton absorption [37].

To stress-test biexciton generation with RAP, we probe a second QD with much larger biexciton binding energy. RAP is more difficult in this case: the pulse area required for efficient $2 X^{0}$ generation increases, potentially entering the regime in which the additional decay process is active. The second QD, embedded in the $n-i-p$ sample [Fig. 1(a)], with biexciton resonance at wavelength $938.2 \mathrm{~nm}$, has a biexciton binding energy of $3.4 \mathrm{meV}$ (the first QD has a binding energy of $1.5 \mathrm{meV}$ ). The $2 X^{0}$ emission intensity of 
(a)

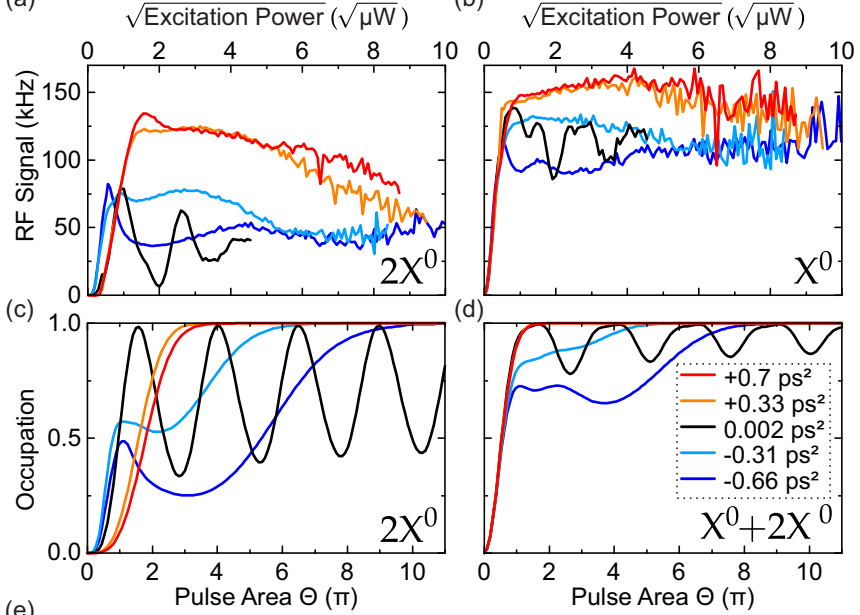

(e)

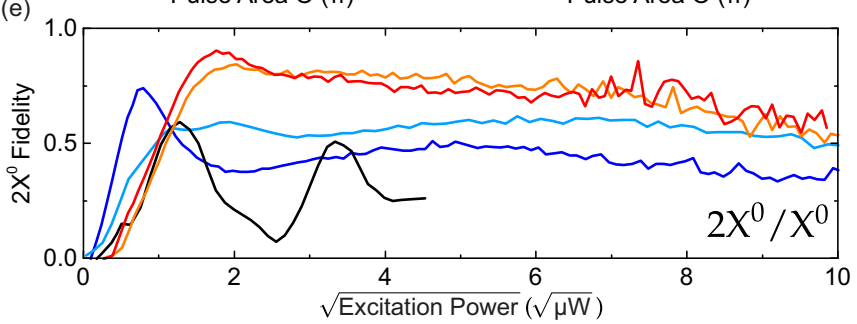

FIG. 3. Biexciton generation on QD2. (a) $2 X^{0}$ emission and (b) $X^{0}$ emission as a function of the time-averaged square-root excitation power for different chirp parameters. (c), (d) Simulation of the biexciton occupation and the sum of exciton and biexciton occupations as a function of the pulse area. (e) Ratio of the experimental data: $2 X^{0} / X^{0}$.

the RAP experiment is shown in Fig. 3. Despite the large biexciton binding energy, the ratio of the $2 X^{0}$ to $X^{0}$ signals reaches $90 \%$ [Fig. 3(e)]. The general behavior is the same as for the first QD: a Rabi rotationlike behavior for minimal chirp and a distinct difference between positive and negative chirp. Again, apart from the decay at high pulse areas, the theory accounts extremely well for the experimental data. Notably, we find that for stronger negative chirp values, the phonons also become more effective resulting in a broader and deeper

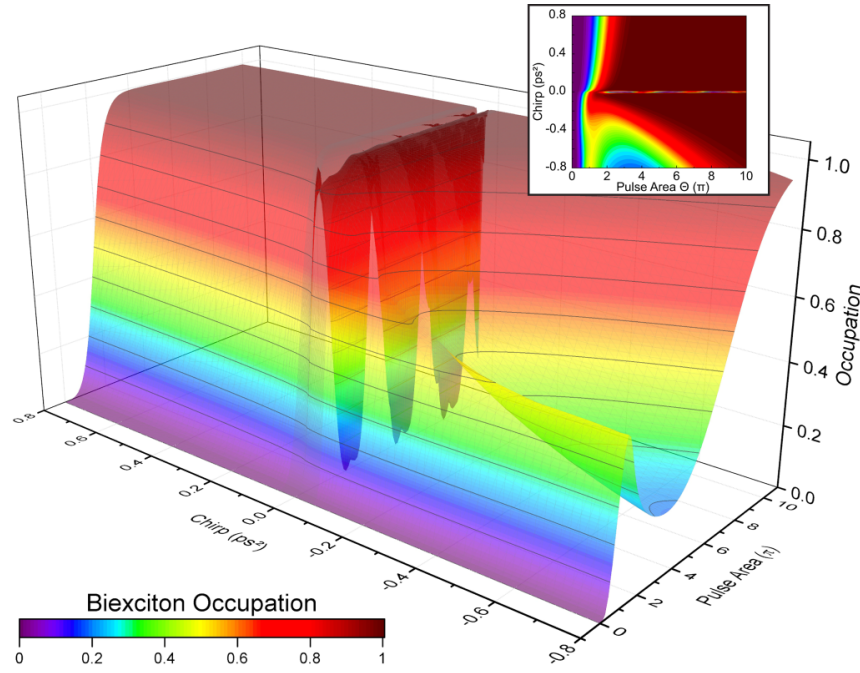

FIG. 4. Calculated biexciton generation on QD2. Simulation of the biexciton occupation as a function of the chirp parameter and the pulse area. The inset shows the top view with the same color scale.

minimum of biexciton occupation which shifts to higher pulse areas (Fig. 4). Further, the RAP onset occurs at smaller pulse areas for negative chirp than for positive chirp (Fig. 4), a feature of the experimental data in Fig. 3(a).

In conclusion, we have demonstrated the coherent generation of a biexciton in a semiconductor quantum dot using a single chirped laser pulse. The state preparation has a very high fidelity over a broad range of excitation powers. The sign of the chirp is important: the scheme is robust with respect to phonon scattering at low temperature for positive chirp. A negative chirp results in damping due to phonon scattering. Theoretical calculations including a microscopic coupling to phonons reproduce all the experimental features apart from a damping in the experiment at high pulse areas.

We acknowledge financial support from EU FP7 ITN $S^{3}$ NANO, NCCR QSIT, and SNF project 200020_156637. A.L. and A.D.W. gratefully acknowledge support from DFH/UFA CDFA05-06, DFG TRR160, and BMBF Q.com-H 16KIS0109.
[1] P. G. Kwiat, K. Mattle, H. Weinfurter, A. Zeilinger, A. V. Sergienko, and Y. Shih, Phys. Rev. Lett. 75, 4337 (1995).

[2] Y.-M. He, Y. He, Y.-J. Wei, D. Wu, M. Atatüre, C. Schneider, S. Höfling, M. Kamp, C.-Y. Lu, and J.-W. Pan, Nat. Nanotechnol. 8, 213 (2013).

[3] N. Gisin, G. Ribordy, W. Tittel, and H. Zbinden, Rev. Mod. Phys. 74, 145 (2002).

[4] M. Müller, S. Bounouar, K. D. Jöns, M. Glässl, and P. Michler, Nat. Photonics 8, 224 (2014).

[5] D. Gammon, E. S. Snow, B. V. Shanabrook, D. S. Katzer, and D. Park, Phys. Rev. Lett. 76, 3005 (1996).

[6] H. Li, Q. Zhuang, Z. Wang, and T. Daniels-Race, J. Appl. Phys. 87, 188 (2000).
[7] G. Juska, V. Dimastrodonato, L. O. Mereni, A. Gocalinska, and E. Pelucchi, Nat. Photonics 7, 527 (2013).

[8] R. Trotta, J. S. Wildmann, E. Zallo, O. G. Schmidt, and A. Rastelli, Nano Lett. 14, 3439 (2014).

[9] K. Kowalik, O. Krebs, A. Lemaître, S. Laurent, P. Senellart, P. Voisin, and J. A. Gaj, Appl. Phys. Lett. 86, 041907 (2005).

[10] M. Bayer, G. Ortner, O. Stern, A. Kuther, A. A. Gorbunov, A. Forchel, P. Hawrylak, S. Fafard, K. Hinzer, T. L. Reinecke, S. N. Walck, J. P. Reithmaier, F. Klopf, and F. Schäfer, Phys. Rev. B 65, 195315 (2002).

[11] R. M. Stevenson, R. J. Young, P. See, D. G. Gevaux, K. Cooper, P. Atkinson, I. Farrer, D. A. Ritchie, and A. J. Shields, Phys. Rev. B 73, 033306 (2006). 
[12] M. Glässl, A. M. Barth, K. Gawarecki, P. Machnikowski, M. D. Croitoru, S. Lüker, D. E. Reiter, T. Kuhn, and V. M. Axt, Phys. Rev. B 87, 085303 (2013).

[13] A. Debnath, C. Meier, B. Chatel, and T. Amand, Phys. Rev. B 88, 201305 (2013).

[14] K. Gawarecki, S. Lüker, D. E. Reiter, T. Kuhn, M. Glässl, V. M. Axt, A. Grodecka-Grad, and P. Machnikowski, Phys. Rev. B 86, 235301 (2012).

[15] H. Y. Hui and R. B. Liu, Phys. Rev. B 78, 155315 (2008).

[16] K. Brunner, G. Abstreiter, G. Böhm, G. Tränkle, and G. Weimann, Phys. Rev. Lett. 73, 1138 (1994).

[17] S. Stufler, P. Machnikowski, P. Ester, M. Bichler, V. M. Axt, T. Kuhn, and A. Zrenner, Phys. Rev. B 73, 125304 (2006).

[18] H. Jayakumar, A. Predojević, T. Huber, T. Kauten, G. S. Solomon, and G. Weihs, Phys. Rev. Lett. 110, 135505 (2013).

[19] H. Gotoh, H. Sanada, H. Yamaguchi, and T. Sogawa, Jpn. J. Appl. Phys. 52, 120202 (2013).

[20] S. Bounouar, M. Müller, A. M. Barth, M. Glässl, V. M. Axt, and P. Michler, Phys. Rev. B 91, 161302 (2015).

[21] P.-L. Ardelt, L. Hanschke, K. A. Fischer, K. Müller, A. Kleinkauf, M. Koller, A. Bechtold, T. Simmet, J. Wierzbowski, H. Riedl, G. Abstreiter, and J. J. Finley, Phys. Rev. B 90, 241404 (2014).

[22] J. H. Quilter, A. J. Brash, F. Liu, M. Glässl, A. M. Barth, V. M. Axt, A. J. Ramsay, M. S. Skolnick, and A. M. Fox, Phys. Rev. Lett. 114, 137401 (2015).

[23] G. Reithmaier, F. Flassig, P. Hasch, S. Lichtmannecker, K. Müller, J. Vučković, R. Gross, M. Kaniber, and J. J. Finley, Appl. Phys. Lett. 105, 081107 (2014).

[24] C.-M. Simon, T. Belhadj, B. Chatel, T. Amand, P. Renucci, A. Lemaître, O. Krebs, P. A. Dalgarno, R. J. Warburton, X. Marie, and B. Urbaszek, Phys. Rev. Lett. 106, 166801 (2011).
[25] Y. Wu, I. M. Piper, M. Ediger, P. Brereton, E. R Schmidgall, P. R. Eastham, M. Hugues, M. Hopkinson, and R. T. Phillips, Phys. Rev. Lett. 106, 067401 (2011).

[26] R. Mathew, E. Dilcher, A. Gamouras, A. Ramachandran, Hong Yi Shi Yang, S. Freisem, D. Deppe, and K. C. Hall, Phys. Rev. B 90, 035316 (2014).

[27] S. Lüker, K. Gawarecki, D. E. Reiter, A. Grodecka-Grad, V. M. Axt, P. Machnikowski, and T. Kuhn, Phys. Rev. B 85, 121302 (2012).

[28] M. Glässl, A. Vagov, S. Lüker, D. E. Reiter, M. D. Croitoru, P. Machnikowski, V. M. Axt, and T. Kuhn, Phys. Rev. B 84, 195311 (2011).

[29] See Supplemental Material at http://link.aps.org/supplemental/ 10.1103/PhysRevB.95.161302 for further information about the set-up, the quantum dot sample and the theory are described in the Supplemental Material.

[30] A. V. Kuhlmann, J. Houel, A. Ludwig, L. Greuter, D. Reuter, A. D. Wieck, M. Poggio, and R. J. Warburton, Nat. Phys. 9, 570 (2013).

[31] O. Martinez, IEEE J. Quantum Electron. 23, 59 (1987).

[32] P. Kambhampati, J. Phys. Chem. Lett. 3, 1182 (2012).

[33] S. L. Sewall, A. Franceschetti, R. R. Cooney, A. Zunger, and P. Kambhampati, Phys. Rev. B 80, 081310 (2009).

[34] D. E. Reiter, T. Kuhn, M. Glässl, and V. M. Axt, J. Phys.: Condens. Matter 26, 423203 (2014).

[35] A. Krügel, V. Axt, T. Kuhn, P. Machnikowski, and A. Vagov, Appl. Phys. B 81, 897 (2005).

[36] A. Kurzmann, A. Ludwig, A. D. Wieck, A. Lorke, and M. Geller, Nano Lett. 16, 3367 (2016).

[37] B. Patton, U. Woggon, and W. Langbein, Phys. Rev. Lett. 95, 266401 (2005). 